Paper \# 104

\title{
Factors Influencing Reinforcement of NR and EPDM Rubbers with Short Aramid Fibers
}

\author{
M. Shirazi, J. W. M. Noordermeer* \\ Elastomer Technology and Engineering Department, University of Twente, \\ 7500AE Enschede, the Netherlands \\ Dutch Polymer Institute DPI, 5612 AB Eindhoven, the Netherlands
}

\author{
Presented at the Fall 178th Technical Meeting of the \\ Rubber Division of the American Chemical Society, Inc. \\ Milwaukee, Wisconsin \\ October 12-14, 2010
}

ISSN: 1547-1977

* Speaker 


\title{
Factors Influencing Reinforcement of NR and EPDM Rubbers with Short Aramid Fibers
}

\author{
M. Shirazi, J. W. M. Noordermeer \\ Elastomer Technology and Engineering Department, University of Twente, \\ 7500AE Enschede, the Netherlands \\ Dutch Polymer Institute DPI, 5612 AB Eindhoven, the Netherlands
}

\begin{abstract}
Among short fiber reinforced composites, those with rubbery matrices have gained great importance due to the advantages they have in processing and low cost, coupled with high strength. These composites combine the elastic behavior of rubbers with strength and stiffness of fibers. Reinforcement with short fibers offers additional features such as design flexibility, high modulus, tear strength, etc. The degree of reinforcement depends on parameters such as: the nature of the rubber matrix, the type of fibers, the concentration and orientation of fibers, fiber to rubber adhesion (generation of a strong interface), fiber length and aspect ratio of the fibers.

In this research aramid fibers have been chosen because of their significantly higher modulus and strength, compared to other commercial fibers. Compounds based on NR and EPDM are prepared. Short aramid fibers with different kinds of surface treatments, standard finish and RFL-coating result in different rubber-fiber interfaces. The reinforcing effect of these short aramid fibers is characterized by mechanical and viscoelastic experiments, and by studying the fracture surfaces with microscopic techniques.
\end{abstract}

\footnotetext{
*j.w.m.noordermeer@utwente.nl; Tel.: 0031-53-4892529; Fax.: 0031-53-4892151
} 


\section{INTRODUCTION}

Fiber reinforced composites with the best mechanical properties are those with continuous fiber reinforcement. Such materials can not be adapted easily to mass production and are generally limited to products in which the property benefits outweigh the cost penalty. ${ }^{1}$ Alternatively, short fibers are used to reinforce polymers in order to improve or modify the thermo-mechanical properties of the matrix for specific applications or to reduce the cost of the fabricated article. ${ }^{2}$ By adding suitable fibers and by controlling factors such as the aspect ratio, the dispersion and orientation of the fibers, and the fiber-matrix adhesion, significant improvements in properties can be achieved with thermoplastic, thermosetting and rubber polymers. ${ }^{1}$

Among different short fiber reinforced composites, those with rubber matrices are gaining increasing importance due to the advantages they impart in processing and low cost coupled with high strength. These composites combine the elastic behavior of rubber with strength and stiffness of the fibers. Moreover, reinforcement with short fibers offers some attractive features such as design flexibility, high modulus, tear strength, etc. Short fiber reinforced rubbers have been successfully used in production of V-belts, hoses, tire treads and complexshaped mechanical goods. ${ }^{3,4}$

Generally, the degree of reinforcement depends upon the nature of the matrix, the type of fibers, the concentration and orientation of the fibers, fiber to rubber adhesion (generation of a strong interface), fiber length and aspect ratio of the 
fibers. ${ }^{3,4,5}$ Poor adhesion increases the critical fiber length, which is the minimum length of fibers needed for effective stress transfer, since mechanical friction at the interface must take the role of adhesion. Good adhesion can nearly double the tensile strength and elongation at break compared to a composite in which the adhesion is poor. ${ }^{6}$

A common method to increase the adhesion is by fiber surface treatment using for example isocyanate or Resorcinol Formaldehyde Latex (RFL); the adhesive treatment for various types of fibers differs. The adhesive layer is applied on a cord by a so-called dipping process. Details of this process can be found elsewhere. ${ }^{7}$

The concept of strength of the interfacial bond is not always clear. In case of perfect adhesion, the matrix or the fiber breaks before the interfacial bond. In absence of adhesion, essentially no work is required to separate the surfaces of the matrix and fiber phases even though the two surfaces may appear to be in contact. However, even in the case of no adhesion, work is required to pull a fiber out of a block of the matrix because of the squeezing force exerted on the fiber as a result of mismatch in coefficient of thermal expansion and cooling down of the composite from the fabrication temperature. Between perfect adhesion and no adhesion there can be many gradations. ${ }^{6}$ In the present work, the reinforcement of two types of widely used rubbers, Ethylene Propylene Diene Rubber (EPDM) and Natural Rubber (NR) is investigated in the form of typical radiator hose and truck tire tread compounds, with short aramid fibers. 


\section{EXPERIMENTAL}

Materials. - Twaron ${ }^{\circledR}$ aramid short fibers were supplied by Teijin Aramid B.V., the Netherlands, with initial length of $3 \mathrm{~mm}$ and fiber diameter of $10-12$ microns. These fibers were chosen because of their significantly higher modulus and strength compared to other commercial fibers. Two types of elastomers have been chosen: Natural Rubber (NR): SMR CV60; and Ethylene Propylene Diene Rubber (EPDM): Keltan ${ }^{\circledR} 8340$ A with 5.5 weight percent ENB content and respectively 55 and 39.8 weight percents of Ethylene and Propylene contents, from DSM Elastomers, the Netherlands. The compositions of the compounds are presented in Table I. Carbon blacks N220 and N550 were obtained from Evonik GmbH (formerly Degussa), Germany. The oil type added to EPDM compounds was Sunpar ${ }^{\circledR} 2280$ paraffinic oil and to NR was naphthenic oil. ZnO was Red Seal quality of Union Minière, Belgium, and stearic acid was a technical quality grade. PolyEthyleneGlycol (PEG2000) was obtained from Merck, Darmstadt, Germany. Sulfur and Tertiary-Butyl-BenzothiazolSulfenamide (TBBS) were provided by Rhein Chemie, Germany. Trimethylolpropane-trimethacrylate (TRIM) and Perkadox 14/40 peroxide were obtained from AkzoNobel, Deventer, the Netherlands. Polymerized 1,2-dihydro-2,2,4-trimethylquinoline (TMQ) came from Flexsys, Belgium.

Two masterbatches were made in a 150 liter industrial internal mixer. The curatives and short fibers were added on a laboratory two roll mill. Composites of the compounds with short aramid fibers, with two different kinds of surface treatment, have been prepared. The different treatments of the fibers were standard finish (StF) which is an oily substance added on the fiber surface to facilitate processing, and Resorcinol Formaldehyde Latex (RFL) coating. It has 
been shown elsewhere that the standard finish has no negative influence on the adhesion of cords to rubbers. ${ }^{8}$

Methods. - The cure characteristics of the compounds were measured with a Rubber Process Analyzer (RPA) of Alpha Technologies, and according to the results the compounds were cured for their t90 +2 minutes in a Wickert hot press WLP1600, at 100 Bar pressure. In order to obtain the preferred orientation of the fibers, before putting into the mold, $20 \mathrm{gr}$ of each compound which was the amount needed to fill the mold, was passed several times through a two roll mill, to make a sheet which could fill the mold. The milling direction was considered as longitudinal direction of fiber orientation.

Tensile tests were done, using a Zwick Z1.0/TH1S tensile tester, speed 200 $\mathrm{mm} / \mathrm{min}$, in the longitudinal direction of fiber orientation on the samples containing each kind of treated fibers, and the fractured surfaces of tensile bars were studied with electron microscopy. Dynamic Mechanical Analysis was done on samples using a Metravib Viscoanalyser DMA +150 , in strain sweep mode, at the frequency of $10 \mathrm{~Hz}$ and ambient temperature.

\section{RESULTS}

Fiber length and dispersion.- Results obtained from studying fiber length and fiber dispersion in model gum stocks, the same compounds but without carbon black and oil, showed that fiber length decreased during mixing. The weight average length obtained for fibers with different treatments in NR and EPDM was approximately between 2.3 to 
$2.7 \mathrm{~mm}$. RFL-treated fibers ended up with higher length in both NR and EPDM after mixing compared to StF-coated fibers.

A dispersion study of the model gum stocks showed that fibers with StF-treatment don't disperse well in NR, tending to form agglomerates of fibers, while they disperse fairly well in EPDM: Figure 1. RFL-treated fibers tend to form smaller agglomerates in both NR and EPDM matrices.

Reinforcement mechanisms and mechanical properties. - Figure 2 shows the tensile test results of the compounds without fibers (WF) and of those containing $5 \mathrm{phr}$ short fibers in longitudinal direction of fiber orientation. Adding fibers causes a drop in elongation at break and tensile strength, as expected ${ }^{9}$, but also results in higher stresses in both low and high strain regimes. Particularly eye-catching is that the reinforcement in sulfur-cured NR (A), especially with RFL-treated fibers is far less than in peroxide-cured EPDM (B). This is highlighted in Figure 3, where the reinforcement factors: the ratio of the stress of a reinforced composite at a certain elongation to the stress of the corresponding compound without fiber at the same elongation, are compared. In the case of NR (A) no large effect of RFL fiber treatment is observed, while for EPDM (B) the effect of the RFL-coating is relatively high, particularly in the range of low elongations till even more than $100 \%$ strain. The tensile stress of peroxidecured EPDM (B) containing RFL-treated fibers increases fast in the beginning, reaching a shoulder, then decreases slightly and later on increases again. This indicates that at the beginning of the tensile test, at low strains, the applied load is mainly transferred to the fibers because of good interaction between peroxide-cured EPDM and the RFLtreated fibers. Apparently, this is not the case for NR. Additional proof for the adhesion of RFL-treated fibers to EPDM (B) compound is that just in that case, SEM pictures of 
the tensile fracture surface show rubber sticking to the fiber surfaces while in other samples no sign of fiber-rubber adhesion was observed: Figure 4.

Considering the improvement in tensile properties of the composites containing fibers treated with standard finish, the increase in stress at both low and high elongations for all composites (A)-(D), and the lack of chemical adhesion between fibers and rubbers in most cases, it is clear that mechanical interaction is of main importance in this fiber reinforcement. The main origins of mechanical interaction have been identified in this study. The first one is roughness of the fiber surface because of fiber bending. Figure 5 shows two pictures of the surface of free standing aramid fibers, in the right picture the fiber is bended/ buckled, and Figure 6 shows a fiber in a tensile fracture surface. The surface becomes rough in bending due to the highly crystalline layer structure of these fibers. Bending/buckling happens a lot of times during mixing, causing this roughness to occur along the contour of the fibers. The second origin of mechanical interaction is fiber ends which have been deformed in the cutting process. Figure 7 shows the end of a bundle of dog-bone shaped short fibers. The diameter of the individual fibers is considerably larger at the ends, caused by the cutting. These end parts can resist pulling out of the rubber matrix by acting as anchors. The third origin of mechanical reinforcement is roughness of the fiber surface due to its coating, important in the case of RFL-treated fibers: Figure 8. This is also a reason that, although no sign of chemical adhesion was observed in sulfur-cured NR (A) compounds, still the compound containing RFL-treated fibers shows slightly better tensile properties compared to the same compound containing standard finish treated fibers.

The effect of curing system.- Two other compounds have also been made: peroxidecured NR (C) and sulfur-cured EPDM (D), to investigate the effect of curing system on 
adhesion: Table I. The tensile curves and the reinforcement factors are also presented in Figures 2, 9 and 10. It appears that with the peroxide curing system for NR (C), RFLtreated fibers show improved tensile properties over sulfur cured NR (A). But particularly eye-catching is the reinforcement achieved with RFL-treated fibers in peroxide-cured EPDM (B) over sulfur-cured (D), where for the latter no sign of chemical adhesion to RFL-treated fibers can be seen in the tensile curve; also the reinforcement factors are very low and close to one compared to peroxide-cured EPDM. In the SEM pictures of tensile fractured surfaces of the two compounds NR (C) and EPDM (D) containing StFas well as RFL-coated fibers, no sign of chemical adhesion was observed either. By comparing Figures 9 and 10 it can be seen that peroxide considerably improves the reinforcing factor in all cases, even for the StF-coated fibers when there is no RFLcoating present.

Dynamic Mechanical Properties. - The results of tan $\delta$ with $\delta$ the loss angle, derived from DMA measurements on compounds sulfur-cured NR (A) and peroxide-cured EPDM (B), are presented in Figure 11. The compounds were loaded with 3 phr fibers instead of $5 \mathrm{phr}$, in order to reduce possible interactions between the short fibers, which might result in a contribution to the measured tan $\delta$. The measurements were done in tension mode, in longitudinal direction of fiber orientation at the ambient temperature. The compounds containing RFL-treated fibers show lower tanס compared to the compounds containing StF-coated fibers. These results are in accordance with the tensile data and show the advantage of RFL-treated fibers, even in the case of no chemical adhesion, over StF-treated fibers in two very common applications: sulfurcured NR and peroxide-cured EPDM compounds. In the case of peroxide-cured EPDM (B), it can be seen that adding StF-coated fibers results in an increase in tanס, because 
of additional loss mechanisms due to slippage on the interface of fiber-rubber. Adding RFL-treated fibers reduces tan delta to almost the same level as for the compound without fibers, because of the chemical bond between fiber and rubber.

It may be expected that, when RFL-treated fibers are added to peroxide-cured EPDM (B), because of the chemical bond between RFL and the bulk rubber matrix and the consequent increase in elastic modulus as a result of reinforcement, the tan $\delta$ could even be lower than for the compound without fibers. The reason that this doesn't happen is that in the production of the short fibers, RFL is applied on cords which consist of bundles of fibers, with the cord being cut later-on to make the short fibers. There is always a considerable fraction of fibers which were in the middle of the bundles that remain uncoated: Figure 12. In the case of sulfur-cured NR (A) there is no considerable change in tanঠ with adding StF-treated fibers. One possible reason can be that because of the much higher modulus of the NR compound compared to EPDM, the contribution of the loss mechanisms at the interface of just 3 phr fibers to tanס of the composite is not significant.

\section{DISCUSSION}

It has become clear that compared to the very common use of RFL-treated textile cords in sulfur cured NR compounds, there are other mechanisms involved in the case of short RFL-treated fiber reinforcement. For RFL-treated cords previous research has shown that in sulfur-cured NR, sulfur and accelerators migrate through the RFL-rubber

interface and create bonds across the phase-boundery. ${ }^{10,11}$ The mechanism proposed was that sulfur forms bonds between the vinyl-pyridine latex in the RFL and the bulk 
rubber. From this perspective it was quite unexpected that the present results indicate a rather poor adhesion between RFL-treated short aramid-fibers and all sulfur-cured rubbers, whether NR or EPDM. This may be due to uneven RFL-coating on the various fibers, as cut out of RFL-treated multi-fiber cords. Even more surprising is that peroxidecuring gives better adhesion than sulfur-curing for all combinations, with RFL- as well as StF-fibers. Most conspicuous is the very good adhesion achieved between RFL-treated fibers and peroxide-cured EPDM. EPDM can well be vulcanized with peroxides and apparently the radicals generated during that process also manage to react with the surface of the untreated StF-fibers, and even more so with the RFL-layer around the others. Whether the latex-particles play here the main role or the resorcinolformaldehyde matrix is at this point not clear and needs further study.

It has been mentioned ${ }^{12,13}$ that RFL loses its adhesion properties with sulfur-cured rubber rather fast when exposed to air, which can be due to oxidation of the latexmoiety in the coating. This oxidation is quoted to be rather fast and happens just within a few days. It could therefore well be conceived that oxidation of the RFL-treated fibers is responsible for the phenomena observed. In our research, no clear sign of chemical bonding between RFL-treated short fibers and sulfur-cured NR was found. The effect of oxidation has also been investigated by using some RFL-coated short fibers which were provided well packed and sealed to prevent oxidation. Using those fibers in NRcompound $(A)$, no difference in tensile properties were found, compared to the same compound with the same amount of RFL treated fibers, stored for quite a long time without particular precautions. Additional proof of no effect of oxidation can be found in the practice in industry: from the moment that RFL-treated cords are purchased from a fiber producing company, till the time that they are used in actual compounds, shipping 
and storage may take several weeks, while the rolls of these cords are not sealed in such a way that no oxygen can reach the cords. Commonly no differences in practical performance are seen in all cases.

\section{CONCLUSIONS}

In the different short fiber / rubber systems examined, clear chemical adhesion just happened in the case of peroxide-cured EPDM with RFL-treated fibers. This phenomenon was most clearly reflected in the tensile curve of this system, showing a large reinforcement factor particularly at tensile elongations of $<100 \%$. But the reinforcement stays in place over the whole range of accessible elongations till break. In addition, peroxide curing has been shown to generally improve interaction between rubber and short aramid fibers, whether with StF or with RFL-treatment. This improvement is probably not always by chemical bonding.

Next to potential chemical bonding, mechanical interaction between fibers and rubber matrix plays an important role. The fibers are long enough to be embedded in a curled manner in the rubber matrix. This impedes pulling out of the rubber matrix, next to surface phenomena on the fibers, as bending/buckling, dog-bone shaped fiber ends and surface roughness due to the RFL-coating. So, even in absence of chemical adhesion, adding short aramid fibers improves the mechanical properties of rubber compounds because of mechanical interaction. 


\section{ACKNOWLEDGEMENTS}

This study is part of the research program of the Dutch Polymer Institute (DPI), under project \# 664. Data on fiber length and fiber dispersion have been provided by Christian Hintze of the "Leibniz Institute of Polymer Research", Dresden, Germany. The Authors gratefully acknowledge Teijin Aramid B.V. and DSM Elastomers B.V. for their support.

\section{REFERENCES}

${ }^{1}$ S. K. De, J. R. White, "Short fiber-polymer composites", Ch. 1, Woodhead publ., Cambridge, England, 1996.

${ }^{2}$ S. Varghese et al., J. Adhesion. Sci. Technol. 8, 235, (1994).

${ }^{3}$ S. Varghese, B. Kuriakose, RUBBER CHEM. TECHNOL. 68, 37, (1995).

${ }^{4}$ H. Ismail et al., Polymer J. Int. 43, 223 (1997).

${ }^{5}$ L. A. Goettler, K. S. Shen, RUBBER CHEM. TECHNOL. 56, 619, (1986).

${ }^{6}$ L. E. Nielson, R. F. Landel, "Mechanical Properties of Polymers and Composites", Ch. 8, $2^{\text {nd }}$ edition, Marcel Dekker Publ., New York, USA, 1994.

${ }^{7}$ D.B. Wooton, "The Application of Textiles in Rubber", Ch. 5, Rapra Publ., Exeter, UK, 2001.

${ }^{8}$ P. J. de Lange et al., J. Adh. Sci. Techn. 23, 139, (2009).

${ }^{9}$ A. Y. Coran, K. Boustany, P. Hamed, RUBBER CHEM. TECHNOL. 47, 369, (1974).

${ }^{10}$ W.B. Wennekes, "Adhesion of RFL-treated cords to rubber", Ph.D. Thesis, University of Twente, Enschede, the Netherlands, 2008. 
${ }^{11}$ W.B. Wennekes, R.N. Datta, J.W.M. Noordermeer, RUBBER CHEM. TECHNOL. 81, 523 (2008).

${ }^{12}$ R. E. Hartz, H. T. Adams, J. Appl. Pol. Sci. 21, 525, (1977).

${ }^{13}$ E. K. Bradley, Rubber and Plastic News, May 1984, p.46-48. 


\section{Captions to the figures}

Fig 1. Dispersion of StF-fibers in gum stocks of NR: left; and of EPDM: right.

Fig 2. Tensile properties of 5 phr fiber-loaded NR- and EPDM-compounds, in longitudinal direction of fiber orientation.

Fig 3. Reinforcement Factor; Black: NR (A); Gray: EPDM (B).

Fig 4. SEM pictures of fibers in tensile fractured surfaces.

Fig 5. Free standing and buckled short aramid fibers (StF).

Fig 6. Short aramid fiber (StF) in tensile fracture surface.

Fig 7. Dog-bone shape fiber ends.

Fig 8. Roughness of RFL-coated short fibers.

Fig 9. Reinforcement Factors; Left: NR, Right: EPDM, containing 5phr RFL-coated fibers, measured in longitudinal direction; Gray: Sulfur-cured, Black: Peroxide-cured.

Fig 10. Reinforcement Factors; Left: NR, Right: EPDM, containing 5phr, StF-treated fibers in longitudinal direction; Gray: Sulfur-cured, Black: Peroxide-cured.

Fig 11. Loss factor tanס derived from strain sweep DMA measurements at ambient temperature for various fiber treatments.

Fig 12. EPDM (B), RFL-treated fibers, partially uncoated. 
Table I: Compound Recipes

\begin{tabular}{lcccc}
\hline Component & A & B & C & D \\
\hline NR & 100 & - & 100 & - \\
EPDM Keltan 8340A & - & 100 & - & 100 \\
Carbon black N-22O & 55 & - & 55 & - \\
Carbon black N-550 & - & 105 & - & 105 \\
Oil & 8 & 60 & 8 & 60 \\
Stearic acid & 2 & 1 & 2 & 2 \\
ZnO & 5 & - & 5 & 5 \\
6PPD & 2 & - & 2 & - \\
TMQ & 1.5 & - & 1.5 & - \\
Wax & 2 & - & 2 & - \\
PEG2000 & - & 2.5 & - & 2.5 \\
TBBS & 1.5 & - & - & 2 \\
Sulfur & 1.5 & - & - & 2 \\
Perkadox 14/40 & - & 7.5 & 7.5 & - \\
TRIM & - & 4 & 4 & - \\
\hline
\end{tabular}



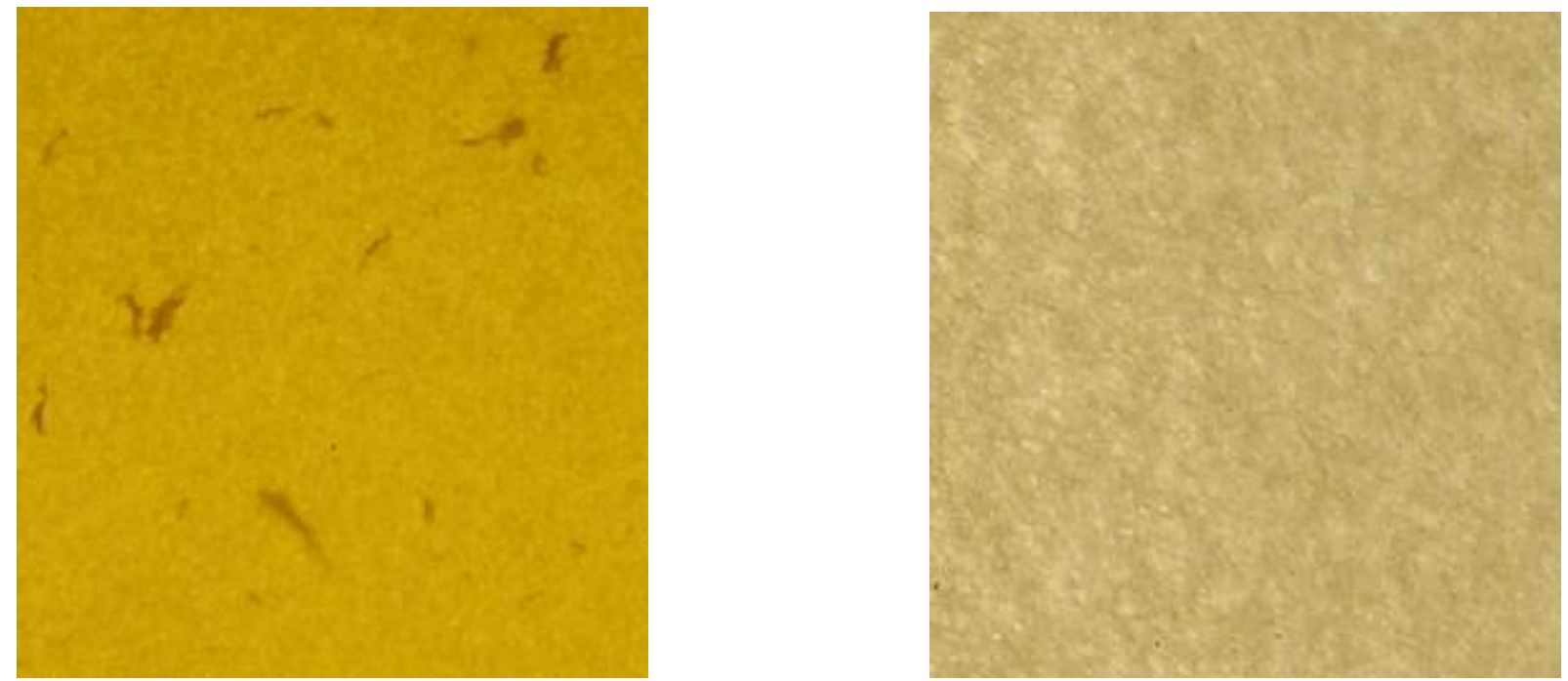

Fig 1. Dispersion of StF-fibers in gum stocks of NR: left; and of EPDM: right. 
NR (A)

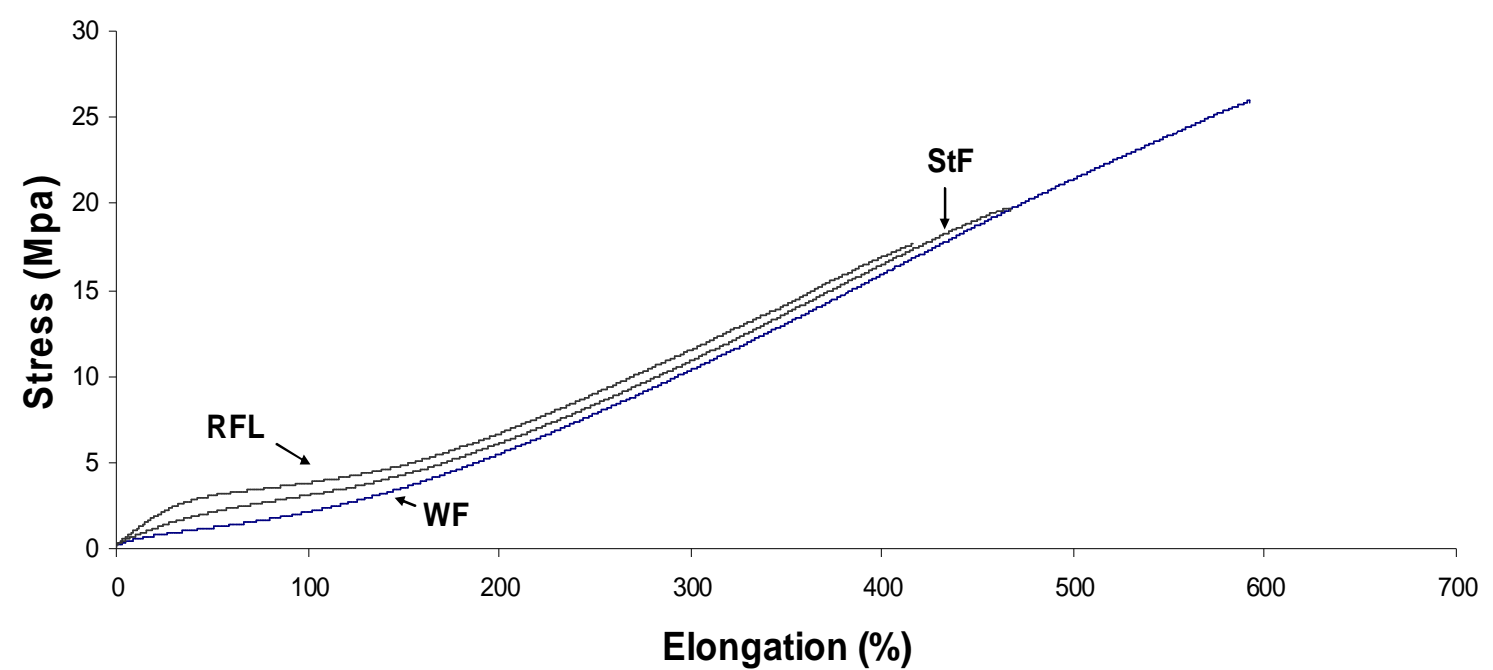

EPDM (B)

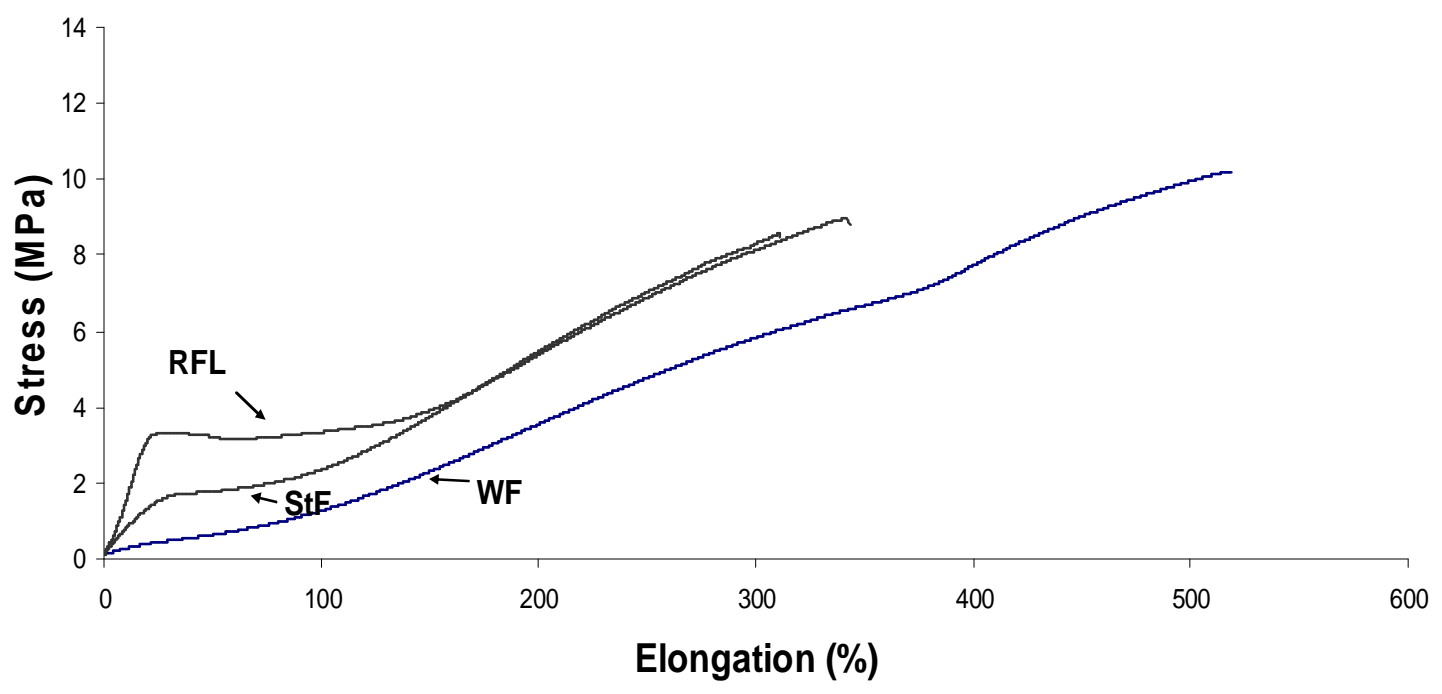


NR (C)

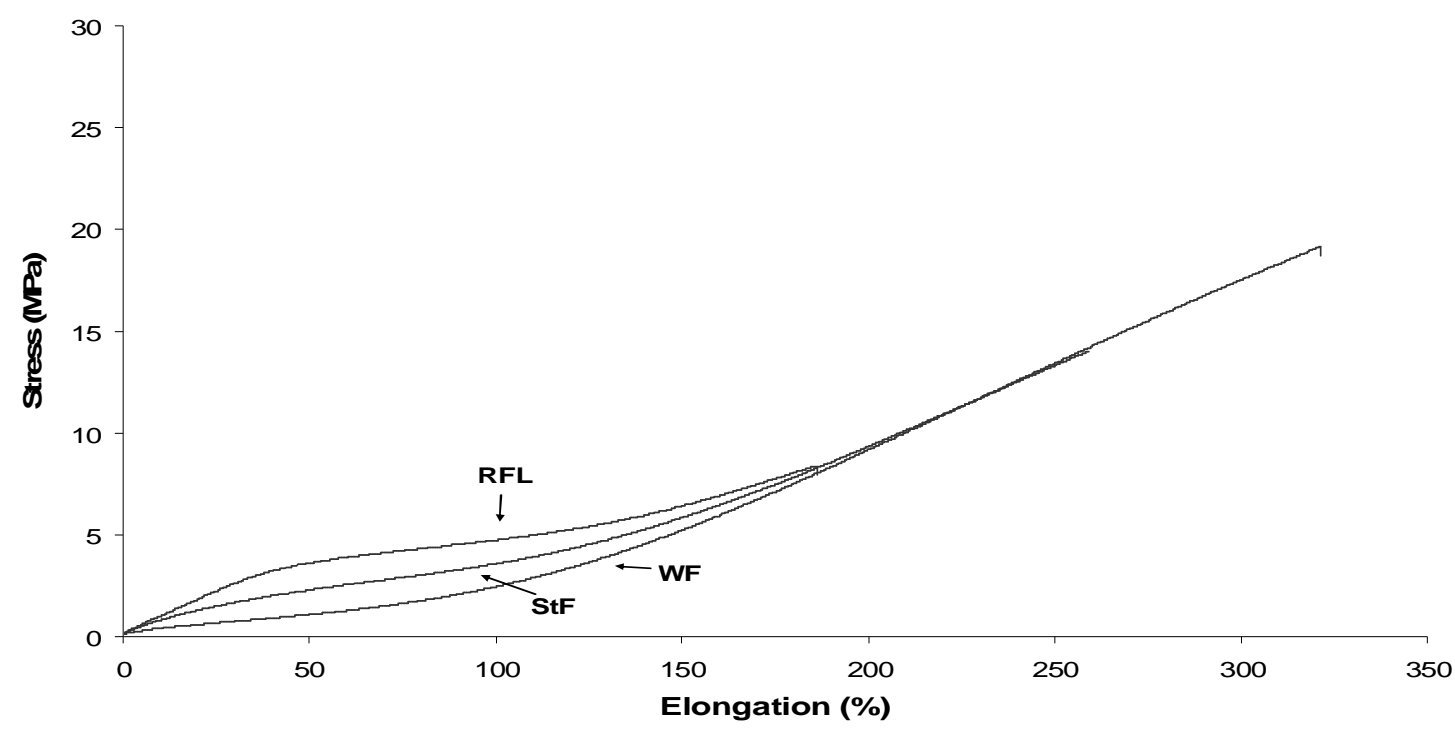

$\operatorname{EPDM}(D)$



Fig 2. Tensile properties of 5 phr fiber-loaded NR- and EPDM-compounds, in longitudinal direction of fiber orientation. 


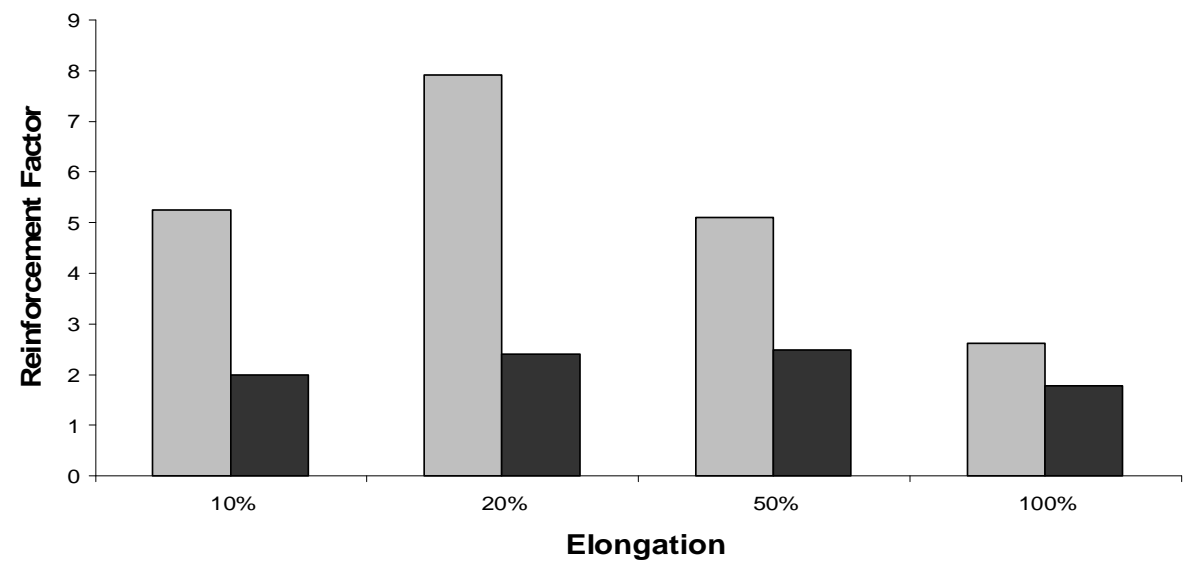

Fig 3. Reinforcement Factor; Black: NR (A); Gray: EPDM (B). 


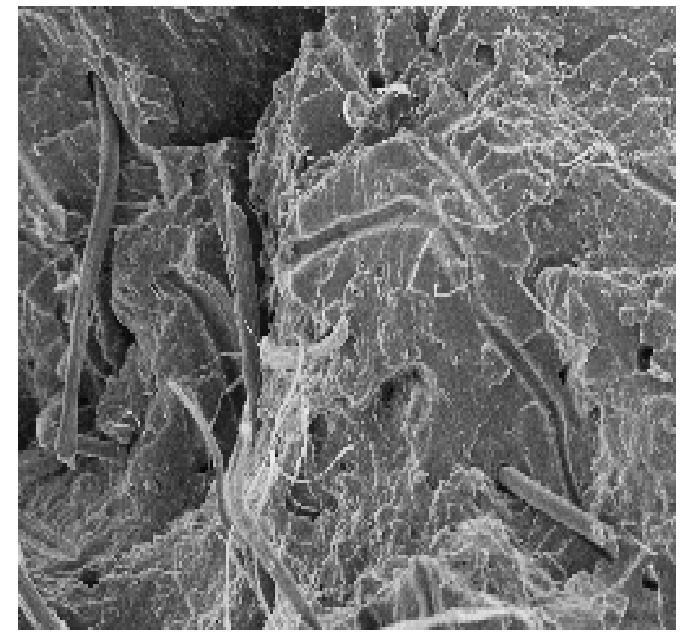

$\mathrm{NR}(\mathrm{A})-\mathrm{StF}$

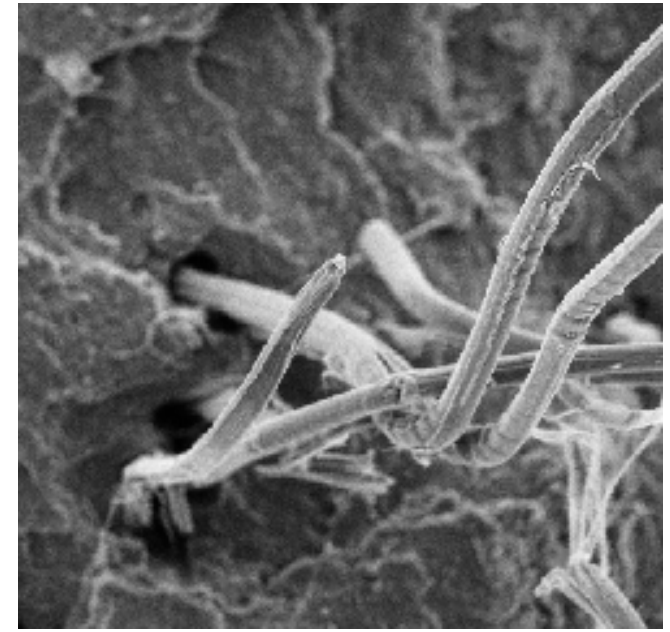

$N R(A)-R F L$



EPDM (B) - STF


$\operatorname{EPDM}(\mathrm{B})-\mathrm{RFL}$

Fig 4. SEM pictures of fibers in tensile fractured surfaces. 



Fig 5. Free standing and buckled short aramid fibers (StF).

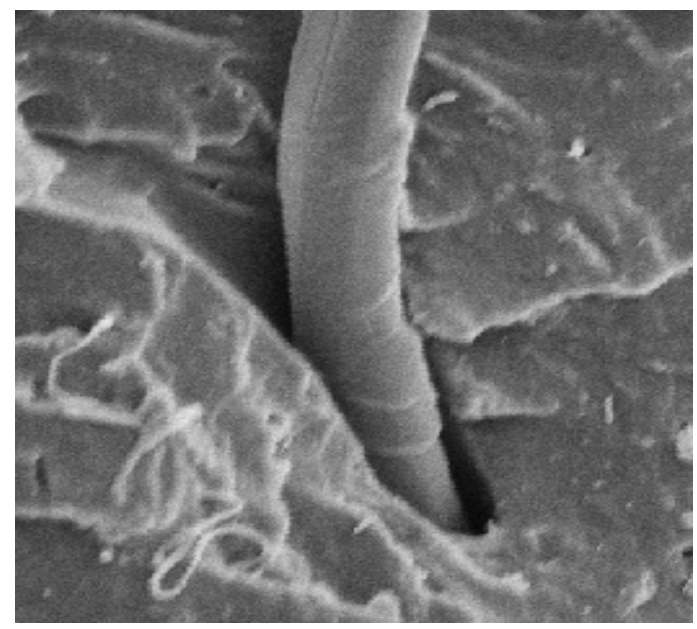

Fig 6. Short aramid fiber (StF) in tensile fracture surface. 


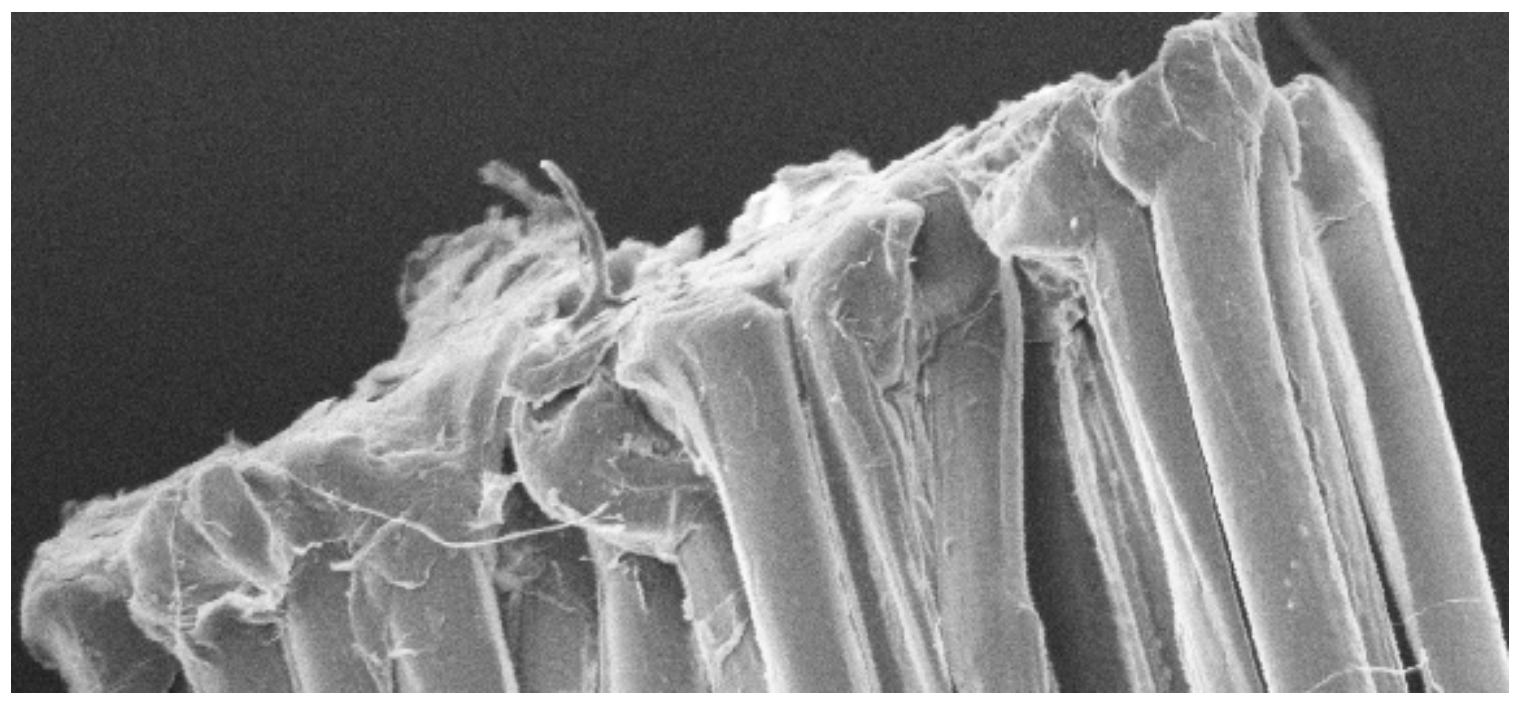

Fig 7. Dog-bone shape fiber ends.

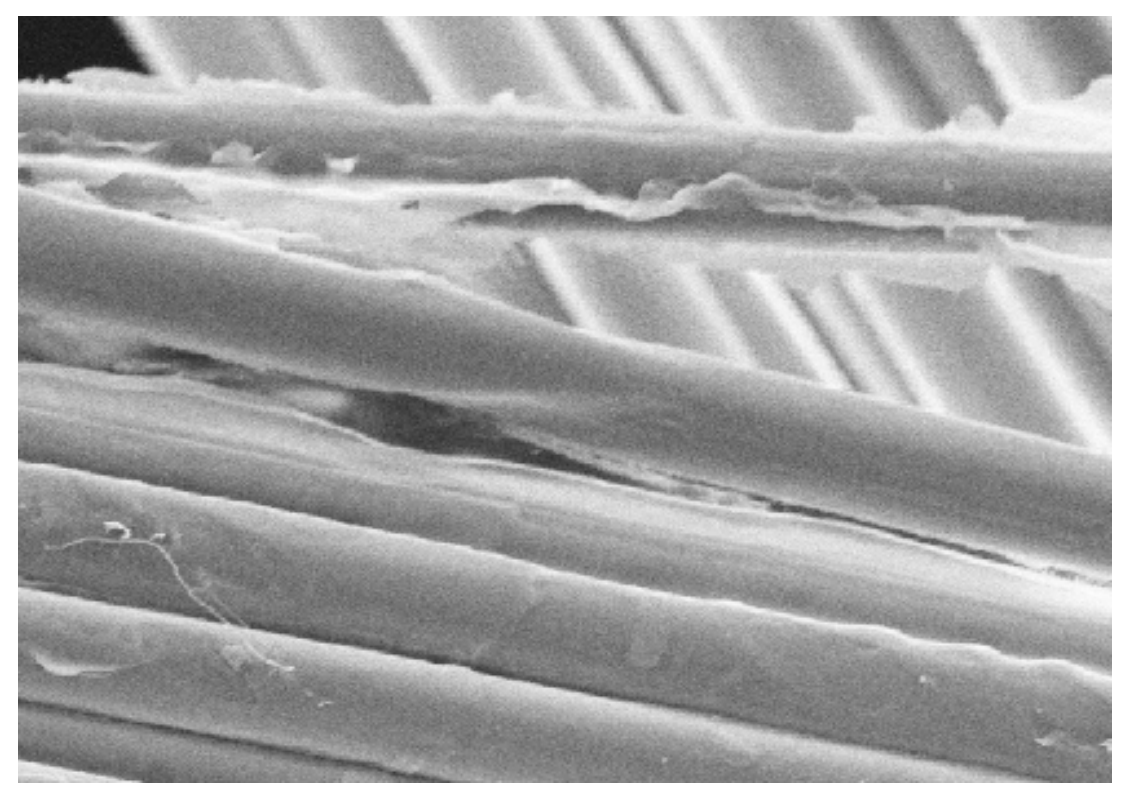

Fig 8. Roughness of RFL-coated short fibers. 

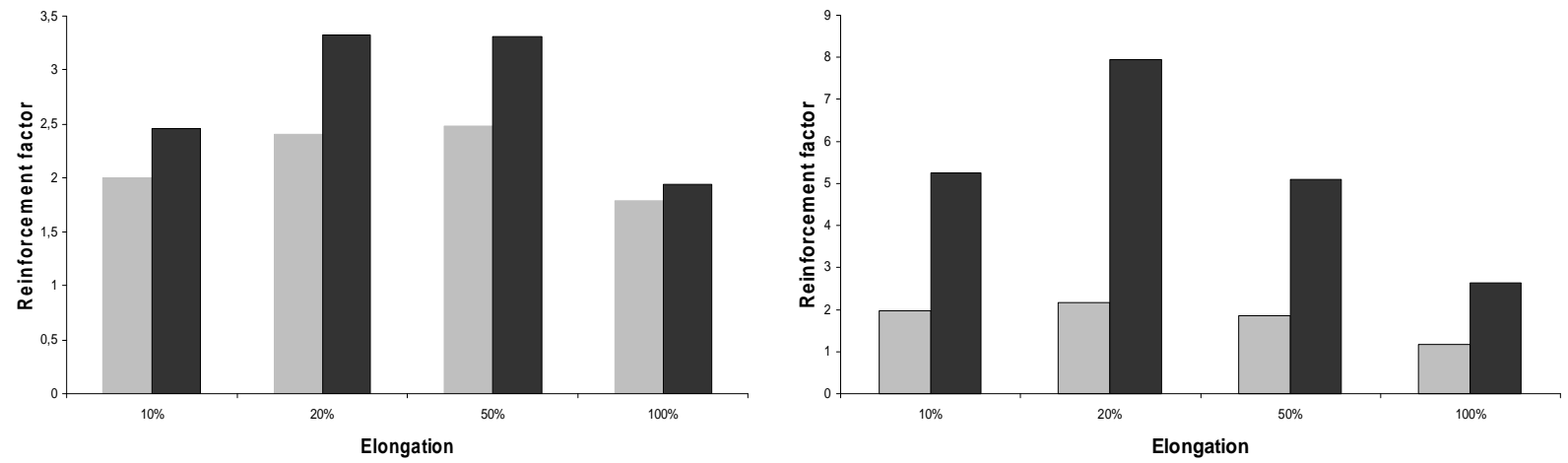

Fig 9. Reinforcement Factors; Left: NR, Right: EPDM, containing 5phr RFL-coated fibers, measured in longitudinal direction; Gray: Sulfur-cured, Black: Peroxide-cured.
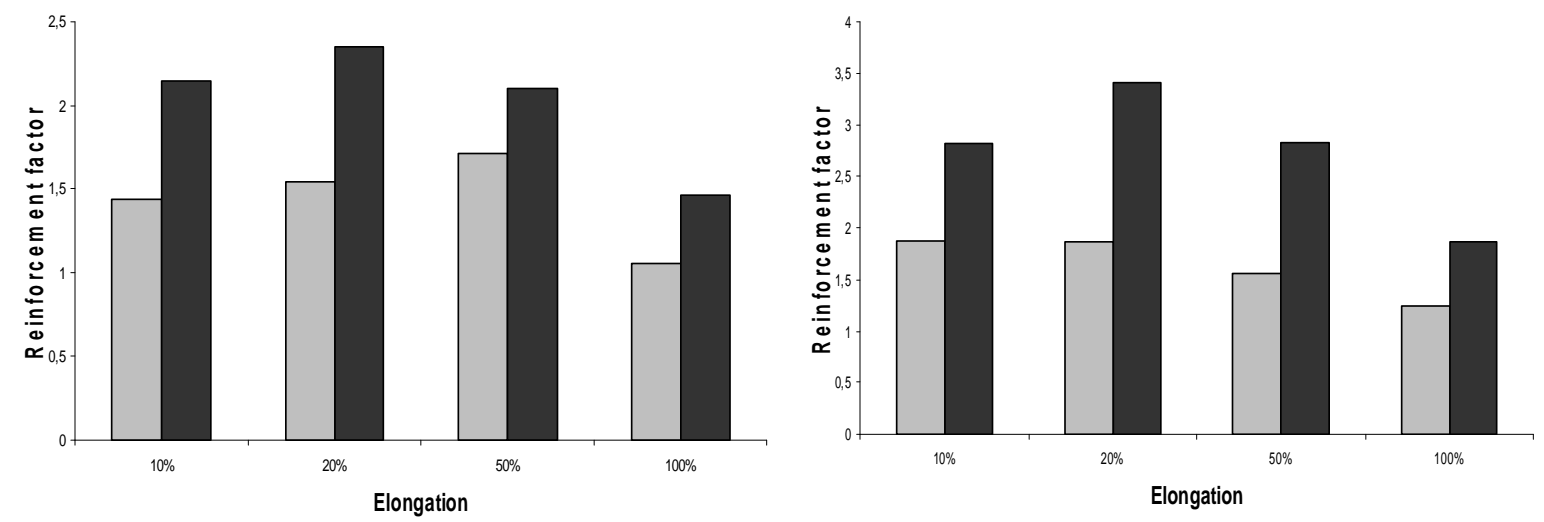

Fig 10. Reinforcement Factors; Left: NR, Right: EPDM, containing 5phr StF-treated fibers in longitudinal direction; Gray: Sulfur-cured, Black: Peroxide-cured. 


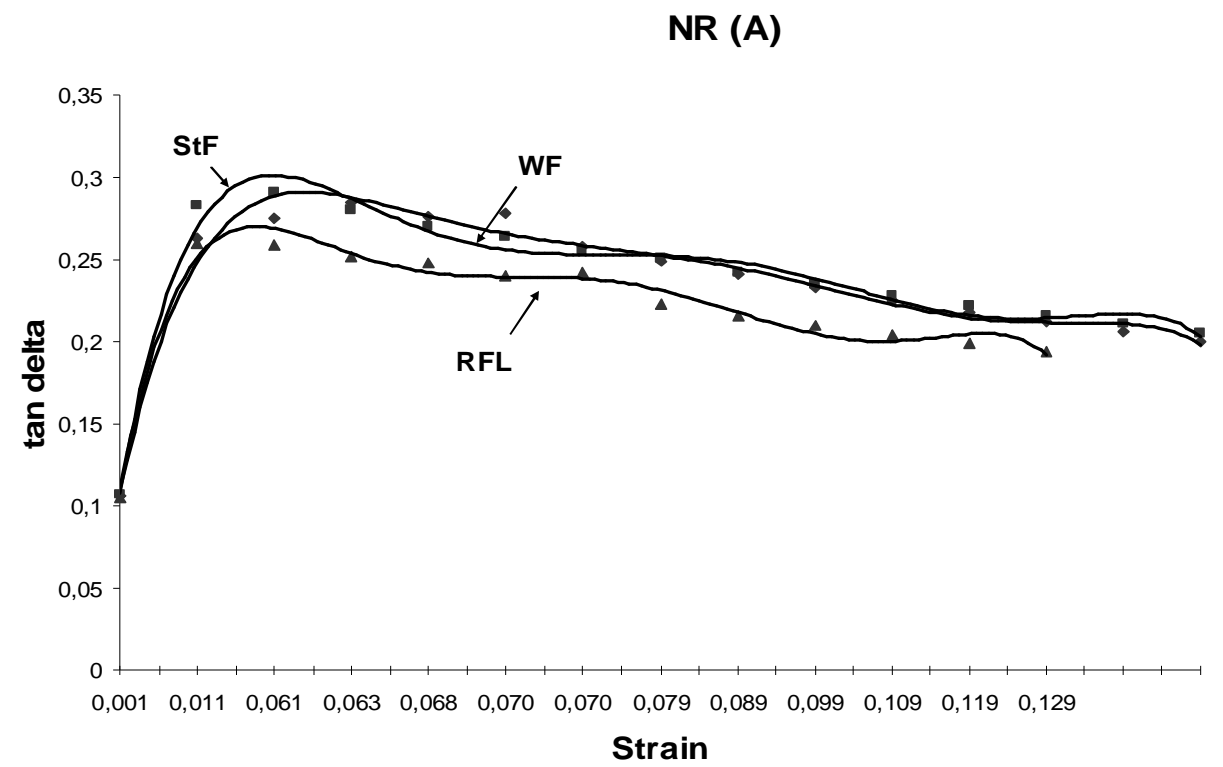

EPDM (B)

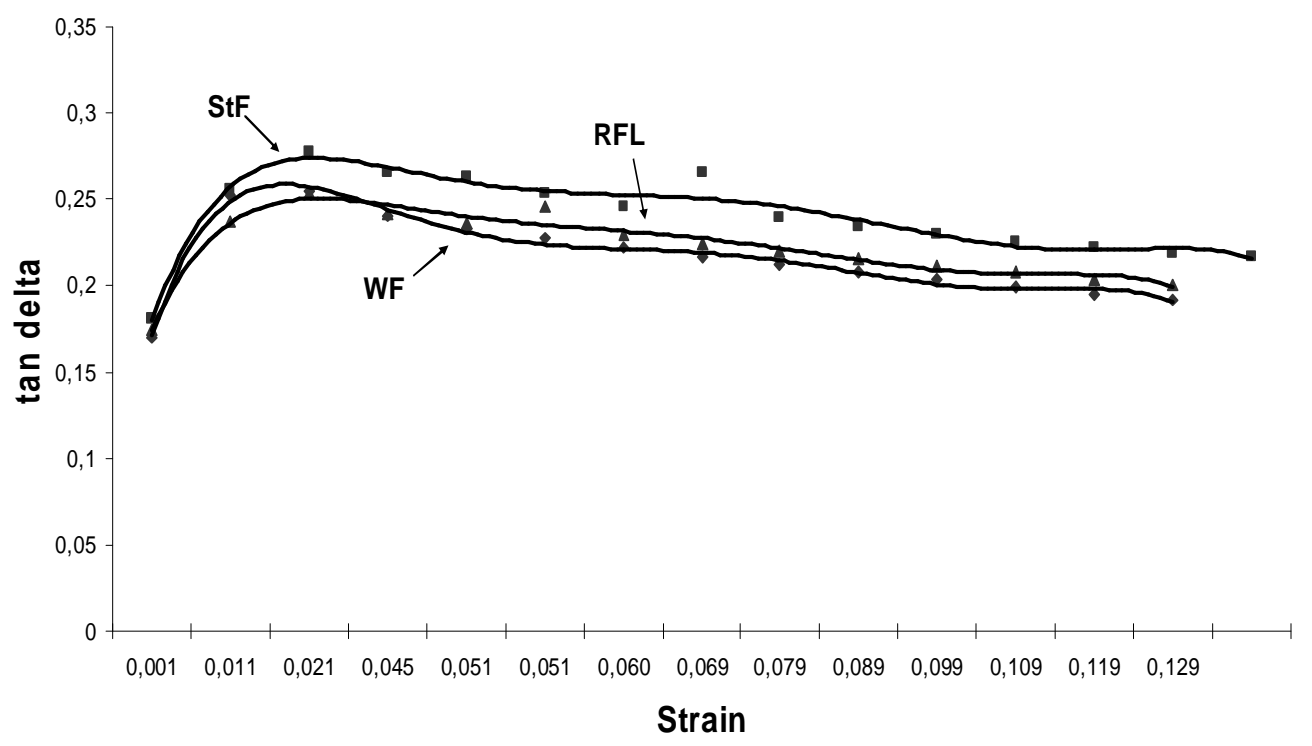

Fig 11. Loss factor tanס derived from strain sweep DMA measurements at ambient temperature for various fiber treatments. 


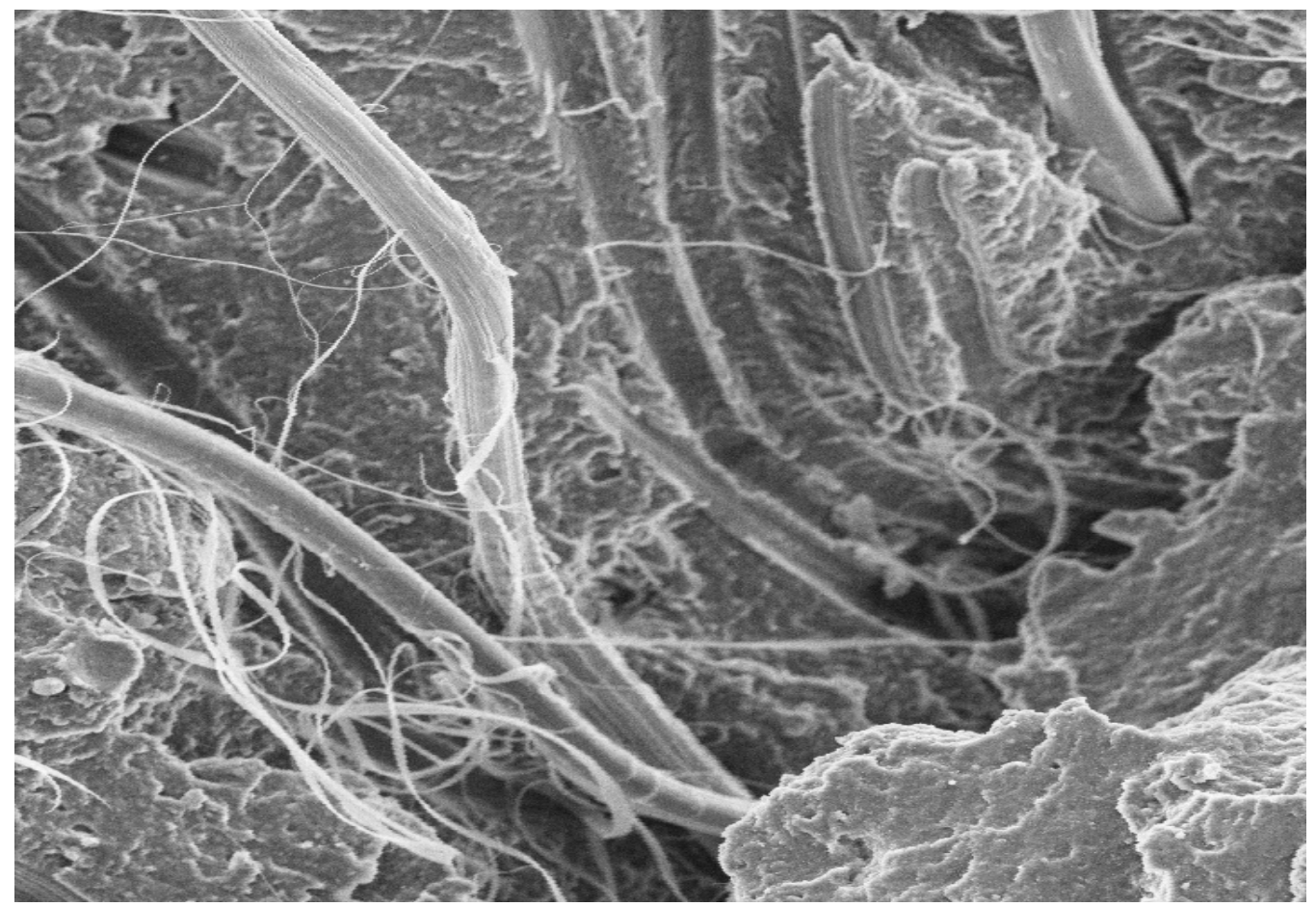

Fig 12. EPDM (B) with RFL-treated fibers, partially uncoated. 